\title{
Ear Recognition with Neural Networks Based on Fisher and Surf Algorithms
}

\author{
Pedro Luis Galdámez, María Angélica González Arrieta, \\ and Miguel Ramón Ramón \\ University of Salamanca, Plaza de los Caídos, 37008 Salamanca, Spain \\ \{peter.galdamez, angelica\}@usal.es, miguel.ramon@dgp.mir.es
}

\begin{abstract}
This paper offers an approach to biometric analysis using ears for recognition. The ear has all the assets that a biometric trait should possess. Because it is a study field in potential growth, this research offers an approach using Speeded Up Robust Features (SURF) and Fisher Linear Discriminant Analysis (LDA) as an input of two neural networks with the purpose to detect and recognize a person by the patterns of its ear. It also includes the development of an application with .net to show experimental results of the applied theory. In the preprocessing task, the system adds sturdiness using Hausdorff distance to increase the performance filtering for the subjects to use in the testing stage of the neural network. To perform this study, we worked with the help of Ávila's police school (Spain), where we built a database with approximately 300 ears. The investigation results shown that the integration of LDA and SURF in neural networks can improve the ear recognition process and provide robustness in changes of illumination and perception.
\end{abstract}

Keywords: Neural Network, Fisher, SURF Algorithm, Ear Recognition.

\section{Introduction}

The ear has been used as a mean of human recognition in forensic activities for long time. During the investigation of several crime scenes, earprints commonly have been used to identify a suspect when there is no information of fingerprints. A recognition system based on images of the ears is very similar to a typical face recognition system, however, the ears have some advantages over the face; for instance, their appearance does not change due its expression is less affected by the aging process; indeed, their details and internal form are maintained over the years, although its size is changing over the years, their color is usually uniform and their background is predictable.

Although the use of information from ear identification of individuals has been studied, it is still debatable whether or not the ear can be considered unique or unique enough to be used as a biometric. However, any physical or behavioural trait can be used as biometric identification mechanism if it is universal, that every human being possesses an identifier, being distinctive and unique to each individual, invariant in time, and measurable automatically or manually; the ear accomplish all these characteristics. 


\section{Brief Review of the Literature}

Significant progress has been made in the past few years in ear biometrics field. One of the most important techniques which are known to detect the ears is raised by Burge and Burger [17] who have made the process of detection using deformable contours with the observation that initialization contour requires user interaction. Therefore, the location of the ear is not fully automatic. Meanwhile Hurley et al. [9] used the technique of force field, this process ensures that it is not required to know the location of the ear to perform recognition. However, only applies when the technique has the specific image of the ear out of noise. In [19], Yan and Bowyer have used manual technique based on two previous lines for detection, where takes a line along the border between the ear and face while another line crosses up and down the ear.

A. Cummings et al. 3] show a strategy using the image ray transform which is capable of highlighting the ear tubular structures. The technique exploits the helix elliptical shape to calculate the localization. Kumar et al [2], have introduced a proposal where uses skin segmentation and edge map detection to find the ear, once they find the ear region apply an active contour technique [20] to get the exact location of ear contours, the technique has been tested over 700 ear images. As well as these techniques there are many other significant proposals.

In other terms a biometric recognition system requires the discovery of unique features that can be measured and compared in order to correctly identify subjects. There are some known techniques for ear recognition specially in $2 \mathrm{D}$ and $3 \mathrm{D}$ images, as the strategies based on appearance, force transformation, geometrical features, and the use of neural networks. The most used technique for face recognition 18, principal component analysis (PCA), is also suitable for use in ear recognition. PCA 12 is an orthogonal transform of a dataset which exploits the training data with the propose to find out a set of orthogonal basis vectors or a new axes that causes the projection onto the first axis (principal component) to represent one greatest variance in data, subsequent orthogonal axes to represent decreasing amounts of variance with minimum reconstruction mean square error. This strategy fall under appearance based techniques.

The first application for ear recognition was the PCA by Victor et al. [4] they used PCA to perform an comparative analysis between face and ear recognition, concluding that the face performs better than the ear. However, Chang et al. [16] also have accomplished a comparison using PCA and found that ears provided similar performance to faces, they concluded that ears are essentially just as good as faces for biometric recognition. There are many proposals to solve the problem, in this paper only has done a small review from some of them, the next section introduce an intent to solve the problem of ear recognition using a practical way, applying some interesting concepts for $2 \mathrm{D}$ images and real time video.

\section{Ear Recognition System}

Most of ear biometric articles have centered their attention on recognition using manually cropped ear images. This is due to the fact that ear detection is a 
complicated problem, especially because ear images vary in pose and scale under different conditions. However, for a robust and efficient ear recognition system is desired to detect the ear from the image face profile in an automatic way.

Recognition systems traditionally follow a set of standards, such as, acquiring images, pre-processing, feature extraction, and the classification. Nevertheless, it is important to notice that the process that we are about to describe is based in the combination of some existing methods in order to build a robust system. In this way, the system combines a series of algorithms that give significant results individually, and when they are combined, achieve a higher degree of robustness with improving in problems such as changes in brightness and perspective.

The chart one shows the workflow that the project will follow; describing how face profile is captured and how we tried to detect the ear. Once it has been detected is extracted removing the background; here the Hausdorff distance is used to filter the candidates, the next step is the feature extraction using SURF and LDA, features are used as input in two neural networks defining a threshold to determine the precision required. If both neural networks compute different results or the result does not exceed the threshold, the system will reject the ear which will be classified as unrecognized. These tasks will be deepened in upcoming sections.

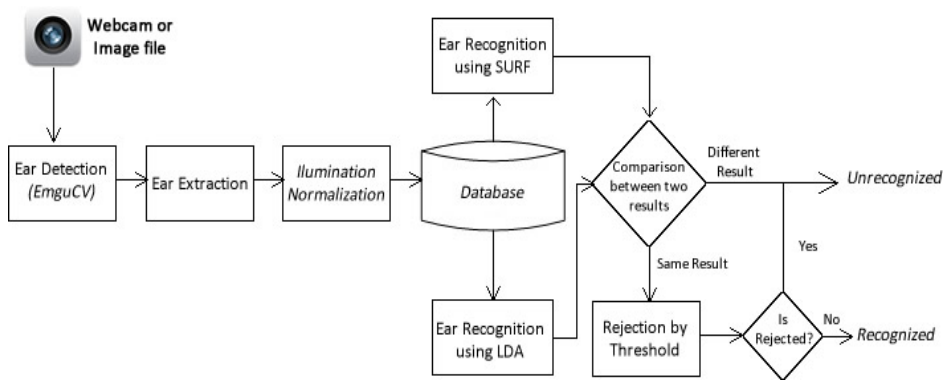

Fig. 1. System flow chart

\section{Detecting and Tracking the Ear}

There are some techniques which could be used to detect ear automatically. In fact, these techniques usually can detect the ear only when a profile face image does not contain a noisy around the ear. These techniques are not useful, when profile face images are affected by scaling and rotation. This section proposes an useful ear localization technique which attempts to solve these issues.

\subsection{Ear Localization}

OpenCV and its wrapper for .net framework EmguCV includes different object detectors based on the Viola-Jones framework, most of them are been constructed to deal with different patterns as frontal face, eyes, nose, etc. Modesto 


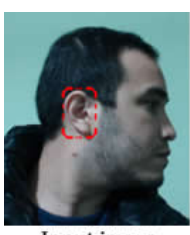

Input image

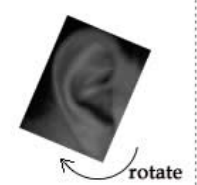

Pre-procesing

Fig. 2. Ear detection

Castellón-Santana et al. 7] have developed a Haarcascade classifier to be used with OpenCV to detect left and right ears. This classifier represents a first step to create a robust ear detection and tracking system. The application is developed in $\mathrm{C \#}$.

With the ear identified we proceed to perform the pre-processing task, converting the image to gray scale and begin the normalization process, the first step is to perform the segmentation of the image applying a mask to extract only the ear, then the image is converted to an edge map using the canny edge filter. If $w$ is the width of the image in pixels and $h$ is the height of the image in pixels, the canny edge detector takes as input an array $w \times h$ of gray values and sigma. The output is a binary image with a value 1 for edge pixels, i.e., the pixel which constitute an edge and a value 0 for all other pixels. We calculate a line between major and minor $y$ value in the edge image to rotate and normalize each image, trying to put the lobule of the ear in the centre. This process is to try to get all the images whose shape is similar to the image to identify. We identify some points on the external shape of the ear and the angle created by the center of the line drawn before and the section in the ear's tragus with the major $x$ value.

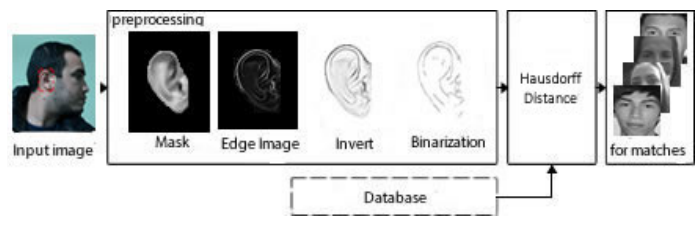

Fig. 3. Image preprocessing

\subsection{Application of the Hausdorff Distance}

The Hausdorff distance measure used in this document is based on the assumption that the ear regions have different degrees of importance, where characteristics such as helix, antihelix, tragus, antitragus, concha, lobe and ear contour; play the most important role in ear recognition. The algorithm applied is based on what is stated in [15. 


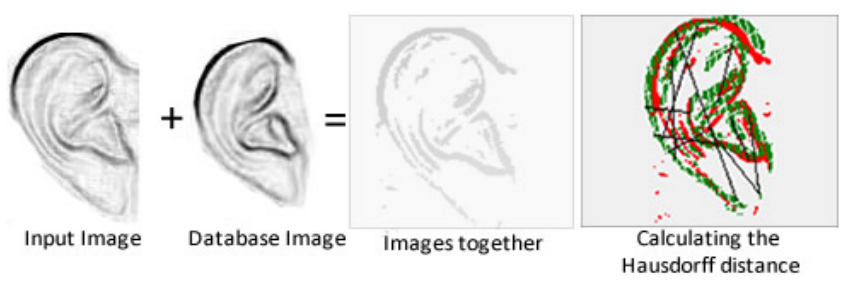

Fig. 4. Hausdorff preprocessing

In applying the Hausdorff distance, basically operates the comparison of edge maps. The advantage of using edges to match two objects, is that this representation is robust to illumination change. Accordingly, the edge detection algorithm used will have a significant effect on performance. Figure 3 shows the flow used in the application of the algorithm, and figure 4 represent an example of the Hausdorff distance trying to put together two images, in this case is not important that two images have been taken in different perspectives because the algorithm try to calculate the distance between the points and with this distance we choose a group of image of our database, this task works like a filter choosing and discarding some images in order to strengthen the classification system.

The procedure involves removing the background of the image as it was performed in the preprocessing original, added some steps after image masking, we proceed to obtain the edges using the canny and sobel filter, the image is reversed to operate with a white background, then the ear is binarized, similar procedure is applied to each image stored in the database. With the obtained objects we compare pixels to get how similar are the two figures, as if they were geometric figures performing a comparison process, calculating the Hausdorff distance, we compare pixels to get how similar are the two figures, resulting in a collection of values that contain the distance of the input image with respect to each item in the database.

The object can be presented as an option having the smaller relative distance; if not exceeds the minimum threshold value and identifies the user, otherwise the problem is considered as an unsolved. In the developed system, the Hausdorff algorithm is presented as an complementary pre-processing task to increase the performance of the neural network and recognition process using SURF algorithm, if the system procedures identify that the user is the same, even without exceeding the thresholds defined in each process, the image is accepted to belong to user input identified by all three techniques combined. In this stage we also compute the SURF features to track the ear in the video.

\subsection{Tracking the Ear}

Speeded Up Robust Features (SURF) 11] is a scale and rotation invariant interest point detector and descriptor. It has been designed for extracting highly distinctive and invariant feature points (also called interest points or key-points) from images. 
One of the basic reasons to use SURF for the feature representation is to analyse how the distinctive characteristics works in images, and at the same time is to found more robust with respect to change, taking into account the point of view, rotation and scale, illumination changes and occlusion [11] as compared to other scale and rotation invariant shape descriptors such as SIFT [8] and GLOH [14. In addition for the extracting SURF features from an image there are two main steps, which describe how to find key points and the calculation of their descriptor vectors. The result for the feature vectors SURF is the relative measured to the dominant orientation to generate each vector that represent an invariant with respect to rotation of the image.

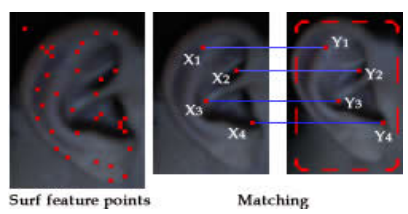

Fig. 5. Example of SURF features

The way SURF process pairing is using the most proximate neighbour ratio pairing. To get the greatest pairing match for a key-point of a picture inside in another picture is elucidated by detecting the most proximate neighbour in the other key-points from a second picture where the most proximate neighbour is defined as the key-point with the least Euclidean distance from the known keypoint of the first picture between their characteristic unidirectional matrices. Due to the fact that these SURF vectors are invariant to the image rotation, the process of ear detection combining the previous viola-jones approach with the SURF vectors becomes robust and efficient.

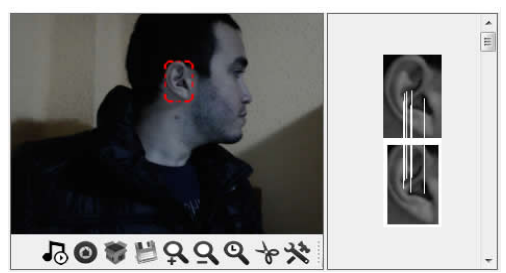

Fig. 6. Tracking ear using SURF features

The approach to isolate the ear in the image, the prototype we used for the ear identification should reveal the characteristics of scale and rotation immutability. To calculate such prototypes in a suggested method, an invariant shape characteristic to rotation and scale was used. Among numerous scale and rotation 
invariant shape characteristics, SURF [13] offers respectable distinctive features and at the same time it is robust to variations in viewing circumstances, rotations and scales. SURF denotes a picture by detecting some exclusive feature points in it and then by describing them with the support of a unidirectional feature descriptor matrix.

\section{$5 \quad$ Ear Recognition Using Neural Networks}

Neural networks provide a great alternative to many other conventional classifiers. This type of algorithms represent powerful tools that can be trained to perform complex tasks and functions in computer vision applications, either in pre-processing tasks, feature extraction and pattern recognition. Two neural networks are used in the system, the first one based on the SURF algorithm we have been talking and the second using a classification based on LDA, both networks have been trained and proven using the database of the police college of Ávila. The training was performed using 3 poses of the ear of each person and the tests were done with $10-n$ poses of the same people.

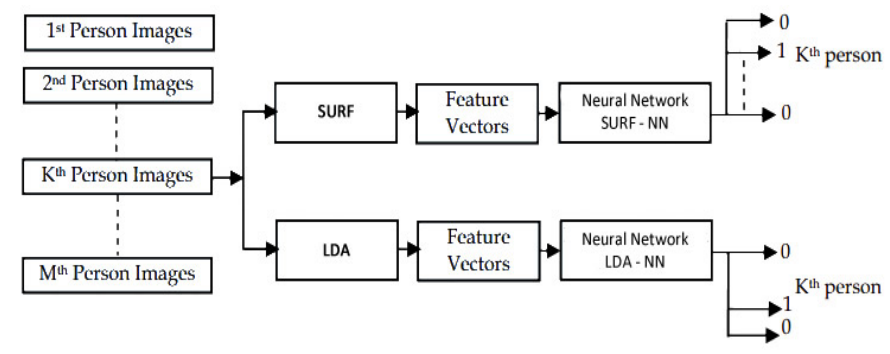

Fig. 7. Training phase of both Neural Networks

After calculating the features using SURF the projection vectors are calculated for the training set and then used to train the network. Similarly, after calculation of the fisherears using the LDA, projection vectors are calculated for the training set. Therefore, the second neural network is trained by these vectors.

\subsection{SURF Neural Network}

The ear image is recreated through the SURF algorithm as a set of salient points, where each on is associated with a vector descriptor. Each can be of 64 or 128 dimensions. If 128 dimensional vector is chosen, it is more exacting in comparison to the 64 vector. So the 128 dimensional descriptor vector is considered the most exacting feature based in the knowledge that is always best to represent the image with the most powerful discriminative features. 


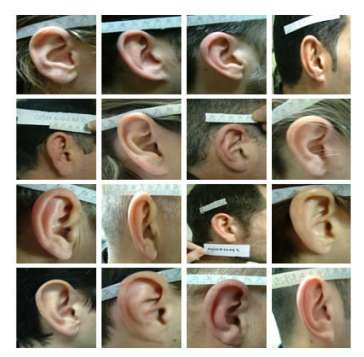

Fig. 8. Avila's Police School Database

A method to obtain unique characteristic fusion of one sole individual is proposed by combining characteristics acquired from various training instances of the individual. If we have $n$ ear images of an individual for training, a fused prototype is gained by fusing the feature descriptor array of all training images collected, considering the redundant descriptor array only once. We had to use a small database made for the training purpose with 309 pictures matching to 3 ear captures from 103 persons. Having all the images processed, a collection was made with their respective tags describing the images and fusion vector calculated before indicating to whom the image belongs.

The parameter settings of the neural network used in this method are dynamic, the output neurons depends on Hausdorff Distance filter stage where the algorithm selects some possible answers to the recognition problem in order to reduce the amount of candidates. The hidden layer is created dynamically, respecting that the number of hidden neurons should be between the size of the input layer and the size of the output layer, should be $2 / 3$ the size of the input layer, plus the size of the output layer; and less than twice the size of the input layer based on the research of Jeff Heaton [10].

\subsection{Linear Discriminant Analysis Neural Network}

Linear discriminant analysis or fisherears method in our case, overcomes the limitations of PCA method by applying the fisher's linear discriminant criterion. This criterion tries to maximize the ratio of the determinant of the between-class scatter matrix of the projected samples to the determinant of the within-class. The LDA approach is similar to the eigenears method which projects the training images into a subspace. The test images are projected into the same subspace and identified using a similarity measure. The ear which has the minimum distance with the test ear image is labelled with the identity of that image. The minimum distance can be calculated using the Euclidian distance. The Fisher algorithm that we implement basically goes like the version exposed in $[5 \mid 21$.

We construct the image matrix $x$ with each column representing an image. Each image is assigned to a class in the corresponding class vector $c$. Then, we proceed to project $x$ into the $(N-c)$ dimensional subspace as $P$ with the rotation matrix WPca identified by a PCA, where: 
- $N$ is the number of samples in $x$.

$-c$ is unique number of classes (length $($ unique $(C)))$

In the next step we calculate the between-classes scatter of the projection $P$ as $S b=\sum_{i=1}^{c} N_{i} *\left(\right.$ mean $_{i}-$ mean $) *\left(\text { mean }_{i}-\text { mean }\right)^{T}$ where:

- mean is the total mean of $P$

- mean $_{i}$ is the mean of class $i$ in $P$

- $N_{i}$ is the number of samples for class $i$

Also, we proceed to calculate the within-classes scatter of $P$ using the next formula $S w=\sum_{i=1}^{c} \sum_{x_{k} \in X_{i}}\left(x_{k}-\right.$ mean $\left._{i}\right) *\left(x_{k}-\text { mean }_{i}\right)^{T}$ where:

$-x_{i}$ are the samples of class $i$

- $x_{k}$ is a sample of $x_{i}$

- mean $_{i}$ is the mean of class $i$ in $P$.

We apply a standard linear discriminant analysis and maximize the ratio of the determinant of between-class scatter and within-class scatter. The solution is given by the set of generalized eigenvectors $W f l d$ of $S b$ and $S w$ corresponding to their eigenvalue. The rank of $S b$ is almost $(c-1)$, so there are only $(c-1)$ non-zero eigenvalues, cut off the rest. Finally we obtain the fisherears by $W=$ $W P c a * W$ fld $[21]$.

These vectors are used as inputs to train our neural network. In the training algorithm, the unidirectional vectors belonging to an individual, are taken as positive returning 1 as the neuron output assigned to that user and 0 to other neurons when the new image has been captured, we compute new descriptors. These descriptors are entered into the neural network, the outputs of individual neurons are compared, and if the maximum output level exceeds the predefined threshold, then it is determined that the user belongs to the ear assigned to the neuron with the index activated.

\section{Experimental Results}

The results obtained in the process of detection and recognition of the ear are presented in this section, table 1 shows the percentages of accuracy when only using the Viola-Jones classifier included in OpenCV vs the potentiation accomplished by adding the tracking with SURF features. That can be seen in 2D images or photographs the difference are not so evident, however when the process is done on video, the difference is almost 10 percentage points, and is only done when considering the location of the ear in the video in different pose and lighting conditions. If we take into consideration the time it succeeds in maintaining trying to identify the object, the algorithm combined with SURF tracking is much more accurate because these features allow you to place the image even if it has a 180 degrees event that does not happen with the ears.

In table 2 and figure 9 we can observe the results of the recognition process and system performance. At this stage we have compared the results obtained 
Table 1. Ear Detection (Haar-Cascade and adding SURF tracking)

\begin{tabular}{|c|c|c|c|}
\hline & \multirow{2}{*}{ \#Attemps } & \multicolumn{2}{|c|}{ EarLocalization $(\%)$} \\
\hline & & Haar-Cascade & acking \\
\hline $\begin{array}{r}2 D \text { Images } \\
\text { Real Time Video }\end{array}$ & $\begin{array}{l}308 \\
314\end{array}$ & $\begin{array}{l}92.53 \\
86.69\end{array}$ & $\begin{array}{l}98.70 \\
95.13\end{array}$ \\
\hline
\end{tabular}

Table 2. (\%)Performance of Conventional PCA vs LDA-NN and SURF-NN

\begin{tabular}{r|rr|rrr}
\hline Training Images & Testing Images & $P C A$ & $L D A-N N$ & $S U R F-N N$ \\
\hline 20 & 80 & 73 & 81 & 82 \\
30 & 71 & 77 & 83 & 84 \\
50 & 87 & 78 & 88 & 84 \\
80 & 104 & 83 & 88 & 89 \\
100 & 149 & 83 & 89 & 93 \\
120 & 186 & 85 & 90 & 94 \\
150 & 305 & 86 & 93 & 97 \\
\hline
\end{tabular}

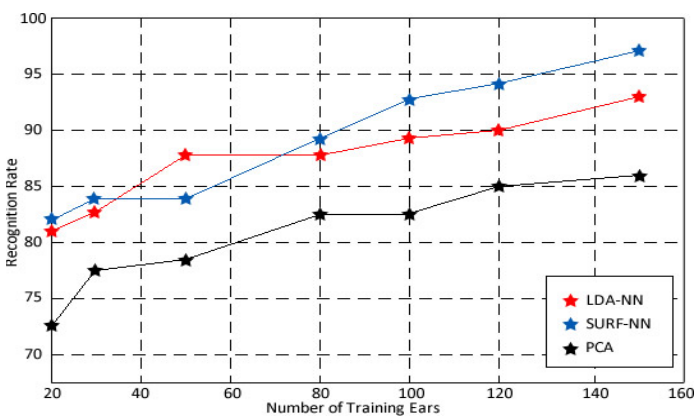

Fig. 9. Recognition rate vs number of training ears

with traditional algorithms such as PCA and our propose using the two neural networks with SURF and LDA to check the validity of our work. In this sense the results are encouraging, using SURF features as input of a neural network with different test subjects, we get a recognition percentage higher than the traditional algorithms in video. Summarizing with perspective and illumination in normal conditions, we get $86 \%$ of succeed in recognition with PCA, 93\% with LDANN algorithm, using the neural network with SURF descriptors, the percentage increased to $97 \%$, over more than 300 attempts of different individuals.

\section{Conclusion and Future Work}

The integration of two algorithms is the main result of this paper. the first technique is based on the SURF preprocessing followed by a feed forward neural network based classifier (SURF-NN), and the second is based on the LDA with 
another feed forward neural network (LDA-NN). The feature projection vectors obtained through the SURF and LDA techniques are used as input values in the training and testing stages in both architectures. The proposed system shows improvement on the recognition rates over the conventional fisher and PCA that use the Euclidean distance. Additionally, the recognition performance of SURF$\mathrm{NN}$ is higher than the LDA-NN among the proposed system.

The neural network using SURF descriptors as input appears to be better over variation in lighting. The LDA-NN and SURF-NN perform better than the PCA traditional method over changes on illumination and perspective. Changes in preprocessing process allows better results specially using Hausdorff distance as a filter stage. Results have shown that approximately $95.03 \%$ of ear recognition accuracy is achieved with a simple 3-layer feed-forward neural network with back-propagation training even if the images contains some noise.

As future work, the most interesting and useful tool for the police is to achieve the development of an application not only able to propose candidates from the image of an ear, but also to achieve the identification and recognition of a criminal using an earprint. The results of this research are pointing towards that goal, they show a significant progress to approach the final purpose, recognition based on these earprints.

Acknowledgements. This work has been carried out by the project Sociedades Humano-Agente: Inmersión, Adaptación y Simulación. TIN2012-36586-C03-03. Ministerio de Economía y Competitividad (Spain). Project co-financed with FEDER funds.

\section{References}

1. Abraham, A.: Special issue: Hybrid approaches for approximate reasoning. Journal of Intelligent and Fuzzy Systems 23(2-3), 41-42 (2012)

2. Kumar, A., Hanmandlu, M., Kuldeep, M., Gupta, H.M.: Automatic ear detection for online biometric applications. In: Proceedings of National Conference on Computer Vision, Pattern Recognition, Image Processing and Graphics, NCVPRIPG 2011, pp. 146-149 (2011)

3. Cummings, A., Nixon, M., Carter, J.: A novel ray analogy for enrolment of ear biometrics. In: Proceedings of International Conference on Biometrics: Theory, Applications and Systems (BTAS 2010), pp. 1-6 (2010)

4. Victor, B., Bowyer, K., Sarkar, S.: An evaluation of face and ear biometrics. In: Proceedings of International Conference on Pattern Recognition (ICPR 2002), vol. 1, pp. 429-432 (2002)

5. Belhumeur, P.N., Hespanha, J.P., Kriegman, D.J.: Eigenfaces vs. Fisherfaces: Recognition Using Class Specific Linear Projection. IEEE Transactions on Pattern Analysis and Machine Intelligence (1997)

6. Borrajo, M.L., Baruque, B., Corchado, E., Bajo, J., Corchado, J.M.: Hybrid neural intelligent system to predict business failure in small-to-medium-size enterprises. International Journal of Neural Systems 21(04), 277-296 
7. Castrillón-Santana, M., Lorenzo-Navarro, J., Hernández-Sosa, D.: An Study on Ear Detection and Its Applications to Face Detection. In: Lozano, J.A., Gámez, J.A., Moreno, J.A. (eds.) CAEPIA 2011. LNCS, vol. 7023, pp. 313-322. Springer, Heidelberg (2011)

8. Lowe, D.G.: Distinctive image features from scale-invariant keypoints. International Journal of Computer Vision 60(2), 91-110 (2004)

9. Hurley, D.J., Nixon, M.S., Carter, J.N.: Force field feature extraction for ear biometrics. In: Computer Vision and Image Understanding (2005)

10. Heaton, J.: Introduction to Neural Networks for C\#, 2nd edn. (2010)

11. Bay, H., Ess, A., Tuytelaars, T., Van Gool, L.: Speeded-up robust features (SURF). In: Computer Vision and Image Understanding (2008)

12. Jolliffe, I.T.: Principal Components Analysis, 2nd edn. Springer, New York (2002)

13. Bustard, J., Nixon, M.: 3D morphable model construction for robust ear and face recognition. In: Proceedings of International Conference on Computer Vision and Pattern Recognition (CVPR 2010), pp. 2582-2589 (2010)

14. Mikolajczyk, K., Schmid, C.: A performance evaluation of local descriptors. IEEE Pattern Analysis and Machine Intelligence (2005)

15. Lin, K.-H., Lam, K.-M., Siu, W.-C.: Spatially eigen-weighted Hausdorff distances for human face recognition, Polytechnic University, Hong Kong (2002)

16. Chang, K., Bowyer, K.W., Sarkar, S., Victor, B.: Comparison and combination of ear and face images in appearance-based biometrics. IEEE Transactions on Pattern Analysis and Machine Intelligence 25(9), 1160-1165 (2003)

17. Burge, M., Burger, W.: Ear biometrics in computer vision. In: Proceedings of International Conference on Pattern Recognition, ICPR 2000 (2000)

18. Turk, M., Pentland, A.: Eigenfaces for recognition. Journal of Cognitive Neuroscience 3(1), 71-86 (1991)

19. Yan, P., Bowyer, K.W.: Empirical evaluation of advanced ear biometrics. In: Conference on Computer Vision and Pattern Recognition-Workshop, vol. 3 (2005)

20. Lankton, S., Tannenbaum, A.: Localizing region-based active contours. IEEE Transactions on Image Processing 17(11), 2029-2039 (2008)

21. Wagner, P.: Fisherfaces (January 13, 2013), http://www.bytefish.de/blog/fisherfaces/ 